\title{
Memory performance, oral comprehension and learning process between children with attention deficit hyperactivity disorder and children with anxiety disorder
}

\author{
Patrícia Aparecida Zuanetti ${ }^{(1)}$ \\ Marília Buzzo Lugli(1) \\ Ângela Cristina Pontes Fernandes ${ }^{(2)}$ \\ Mônica da Silva Trabuco Soares ${ }^{(1)}$ \\ Kelly da Silva(3) \\ Marisa Tomoe Hebihara Fukuda ${ }^{(4)}$
}

\section{(1) Hospital das Clínicas da Faculdade de Medicina de Ribeirão Preto da Universidade de São Paulo, Ribeirão Preto, São Paulo, Brasil. \\ (2) Universidade Paulista, Ribeirão Preto, São Paulo, Brasil. \\ (3) Universidade Federal de Sergipe, Lagarto, Sergipe, Brasil. \\ (4) Faculdade de Medicina de Ribeirão Preto da Universidade de São Paulo, Ribeirão Preto, São Paulo, Brasil. \\ Research carried out at Faculdade de Medicina de Ribeirão Preto, Universidade de São Paulo (FMRP-USP), Ribeirão Preto, São Paulo, Brasil. \\ Research support source: Fundação de Amparo a Ensina, Pesquisa e Assistência do HCRP - USP (FAEPA)}

Conflito de interesses: Inexistente

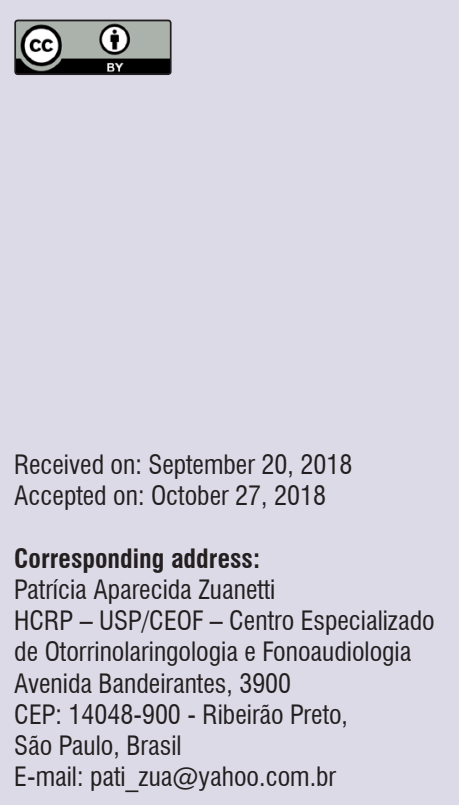

\section{ABSTRACT}

Purpose: to compare aspects of memory, learning and oral comprehension between children with Attention Deficit Hyperactivity Disorder (ADHD) and children with Anxiety Disorder (AD).

Methods: thirty-two children (7-10 years) were divided into: G1 - children diagnosed with ADHD, and G2 - children diagnosed with AD. The children were not under drug treatment. The tests applied assessed working memory (phonological loop and visuospatial sketch), learning, episodic memory and oral comprehension.

Results: both groups showed changes in working memory for visuospatial sketch and phonological loop (worse performance in pseudowords in the ADHD group and digit-reversed order for children with AD), and in oral comprehension. Group comparison showed a statistically significant difference regarding the most complex level of the oral comprehension test and the repetition of nonwords with three syllables. Both groups showed a suitable performance in learning ability, however, the group of children with ADHD suffered from backward interference, with no memory consolidation, showing low episodic memory performance.

Conclusion: children with ADHD and anxiety disorder showed various altered cognitive skills, although group comparison revealed that children with ADHD exhibited worse cognitive performance.

Keywords: Memory; Learning; Comprehension; Anxiety Disorders; Attention Deficit Disorder with Hyperactivity; Child Language 


\section{INTRODUCTION}

The prevalence of children with difficulties in some academic domains is alarming. A Brazilian study ${ }^{1}$ has shown that approximately $55 \%$ of children of school age (1st to 5th year of Elementary School) exhibited inferior performance in the Portuguese language and/ or mathematics. This group of children contains those diagnosed with specific learning disorders (SLD) such as dyslexia and children diagnosed with secondary learning disorders. The changes in school domains are due to some other condition such as intellectual deficiency, abuse and/or mood disorders and others ${ }^{2}$. In the present national study ${ }^{1}$ the two most prevalent conditions among children with altered school performance were Attention Deficit and Hyperactivity Disorder (ADHD) and Anxiety Disorder (approximately 55\%), exceeding the number of children with specific learning disorders.

ADHD is considered to be a disorder of neurodevelopment, i.e., a condition that starts during infancy. The behavioral manifestations involved in neurodevelopmental disorders are quite varied and involve cognitive, linguistic, behavioral and social impairment ranging from very specific limitations to global impairment ${ }^{2}$. Specifically, the essential characteristic of ADHD is a persistent pattern of disattention and/or hyperactivity/ impulsivity that interferes with the functioning/routine of the child. Disattention behavior is associated with various underlying cognitive processes, and persons with ADHD may exhibit cognitive problems in attention, executive function or memory tests, although these tests are not sufficiently sensitive or specific to be used as diagnostic indices. Unsatisfactory school performance is cited as the main impairment during school age as a consequence of the cognitive changes present in this population ${ }^{2}$.

Regarding $A D$, the estimate is that up to $10 \%$ of the child population may exhibit some pathological signs and symptoms of anxiety ${ }^{3}$. The basis of this disorder consists of characteristics of excessive fear and anxiety and behavioral disorders ${ }^{2}$. Anxiety disorders differ from adaptive fear or anxiety by being excessive or by persisting beyond periods appropriate for the developmental level of the subject. During cildhood, the most prevalent subtype is separation anxiety ${ }^{3}$, with a reduction in the prevalence of this type of anxiety ocurring from childhood to adolescence and adulthood $^{2}$. Specific or generalized phobias are also common during childhood².
International and Brazilian studies ${ }^{4-12}$ have shown that children with ADHD or attention disorder (AD) are cognitively inferior to control children, showing difficulties of memory ${ }^{4-10}$, attention ${ }^{8,11}$ and oral comprehension ${ }^{12}$, although few studies have compared the cognitive performance of children wit ADHD and $A D$. Since these two disorders are frequent during childhood and cause changes in the learning process of academic domains, future studies are needed to compare the cognitive performance of these two groups, with details about which of the two conditions involves a greater cognitive impairment and which differences in performance exist between them in order to look for possible diagnostic indicators.

The type of memory most extensively studied in the child population, related to executive function and oral and written language development, is working memory (WM). Currently, the best known WM model is multicomponent WM. This is short-term memory involved in the manipulation of information in order to permit the execution of complex cognitive tasks ${ }^{13}$.

In this multicomponent model, WM consists of four components: the executive central one (whose function is to focus attention on the task and to switch attention between two or more tasks, among others), the phonologic loop (which maintains information verbally coded), the visuospatial binding (which is responsible for the processing and maintenance of visual and spatial information), and the episodic buffer (the system responsible for the process of integration of the information maintained in the various subcomponents of WM with that maintained in the long-term memory) ${ }^{13}$. Within the clinical context, the subcomponents most extensively evaluated are the phonologic loop, by means of digit repetition (digit span), words and nonwords, and the visuospatial memory (Corsi blocks).

Oral comprehension is one of the tasks assessed within language evaluation - receptive sphere. The token test instrument, short version is frequently used to assess language within the context of neuropsychological evaluation. The test permits to assess oral comprehension by means of simple commands dictated by the examiner ${ }^{14}$. The original version of this test was presented by De Renzi and Vignolo in the early sixties and was followed by various adaptations. The short version was presented by the same authors in $1975^{15}$.

Learning ability, recent and recognition memory, information retention and susceptibility to interference are skills that can be assessed with the Rey 
Auditory-Verbal Test (RAVLT), an extensively used instrument for neuropsychological evaluation of both child and elderly populations in various countries. The definition of recent memory is related to working memory - phonologic loop as described earlier. Learning skill correponds to how much a subject is able to memorize items (learn) after each new attempt, i.e., how many more stimuli he remembers after training. Susceptibility to interference corresponds to how much new stimuli impair the ability to remember old stimuli, and finally recognition memory is the identification and differentiation between a previously exposed content and a new content ${ }^{16}$.

The above skills are the cognitive skills assessed in a neuropsychological evaluation that aims to understand the cognitive functioning and the interfaces between emotional and social aspects. In 2015, the Federal Council of Phonoaudiology issued rules about the attributions and competences of Speech Therapists Specialized in Neuropsychology, stating that these professionals are able to assess brain functioning and its interface with linguistic alterations ${ }^{17}$.

Since $A D H D$ and $A D$ are frequent disorders in the child population both characterized by cognitive changes and inferior school performance, and since a speech therapist usually is one of the first professionals receiving these children and is also able to assess cognitive functioning, it is necessary for the professional speech therapist to better understand the cognitive performance of these two populations, being able to identify the differences in behavior and in cognitive performance of these children. Thus, the objective of the present study was to compare the aspects of memory, learning and oral comprehension of children with an ADHD diagnosis and children wih an $A D$ diagnosis.

\section{METHODS}

This retrospective study was analyzed and approved by the Ethics Committee for Research on Human Beings of the University Hospital, Faculty of Medicine of Ribeirão Preto, University of São Paulo (approval protocol no. 12346/201). In agreement with ethical questions, since this was a study on data referring to a health care service and belonging to a protocol of service evaluation, there was no need to obtain written informed consent.

Thirty-two children participated in the study and were divided into two groups.
- Group 1 (G1): 17 children with a diagnosis of Attention Deficit and/or Hyperactivity Disorder (53\% - 9 children had the disattention subtype and the remaining ones had the combined type). Mean \pm SD age was $9 \pm 1.62$ years; $41 \%$ (7) of the children were girls.

- Group 2 (G2): 15 children with a diagnosis of Anxiety Disorder $(60 \%$ - 9 children - had separation anxiety disorder). Mean \pm SD age was $9.4 \pm 1.29$ years; $53 \%$ (8) of the children were girls.

The study sample was obtained by analyzing the medical records of 115 children submitted neuropsychological and speech-language assessment and monitored by multidisciplinary teams (neurologist and/ or psychiatrist, psychologist and speech-language therapist) of a tertiary hospital from April 2015 to July 2017. The main objective of the outpatient clinics where the children were followed up was the treatment of $A D H D$ and of internalizing disorders or learning disorders. The data for 83 children were excluded from this initial sample, mainly owing to the "use of medication" variable at the time of speech-language and neuropsychological assessment.

Inclusion criteria were: absnence of hearing loss of some type or degree, intellectual estimate classified as medium-inferior, medium or medium-superior; absence of syndromes or of other diseases that impair cognitive capacity (e.g.: fetal alcoholic syndrome, intellectual deficiency, among others); absence of medication for the treatment of ADHD and mood disorders in the last six months preceding the neuropsychological and speech-language evaluation; time of less than one month between the neuropsychological and speechlanguage assessment. Children with a diagnosis of Attention Deficit Disorder and/or Hyperactivity (of either the combined or disattention type) were included in Group 1, and children with a diagnosis of Anxiety Disorder regardless of type (separation, social phobia etc.) were included in Group 2.

Exclusion criteria were: presence of a comorbid diagnosis of ADHD and mood disorder (depression and/or anxiety), a comorbid diagnosis of specific learning disorder and ADHD or mood disorder, and age of less than seven years or more than 10 years (an age for which there is no normality standard for the instrument used in the present study).

After subject selection, data were collected from specific reports for each area (neuropsychology and speech-language therapy), together with data 
regarding diagnosis, medical monitoring and medical history.

The instruments used in the present study are listed below:

\section{Rey Auditory-Verbal Learning Test (RAVLT)}

This test assesses memory aspects by means of verbal stimuli, as well as learning ability. In the present study, we used the test constructed by Oliveira and Charchat-Fichman ${ }^{18}$ which is based on the RAVI paradigm. The RAVLT constructed by these authors is a more appropriate version for Brazilian children and provides results similar to those of the traditional RAVLT.

This test consists of a list of 12 nouns (A list) which is read aloud four consecutive times to the subject with one-second intervals between words ( $A 1$ to $A 4$ ). Each attempt is followed by a spontaneous evocation test. After the fourth attempt, an interference list also consisting of 12 nouns (B list) is read to the subject and is followed by list evocation (B1). Immediately after the $B 1$ attempt, the subject is asked to remember the words of the A list without a new repetition (A5). After a 30 minute interval, the subject is asked to remember the words of the A list (A6) again without repetition. After the $A 6$ attempt, the recognition list test (recognition memory) is applied, when a list containing 12 words from list $A, 12$ words from list $B$ and other distractors (similar to the words of the A and $B$ lists in phonologic or semantic terms) is read to the subject. For each word read, the subject must indicate if it belongs (or not) to list $A$.

For statistical analysis, the children were classified according to age for the parameters related to immediate memory (A1), learning curve for the words along the attempts (sum of the words spoken from arttempt $A 1$ to $A 4)$, learning rate (A4 - A1), retroactive interference index (A5/A4) which assesses the interference of a new content with the learning of a previously learned content, the ability to retrieve (A6 - long-term memory), and the recognition item (recognition memory).

\section{Nonword and Digit Memory Test ${ }^{19}$}

A test that assesses working memory using auditory clues. The test consists of three tasks: nonword repetition, direct order digit repetition and backward digit repetition.
The nonword repetition task consists of 40 nonwords of two to four syllables. The subject scores 2 points when he repeats correctly the first time and 1 point when he repeats correctly the second time. Digit recall is assessed in the direct and backward recall order using two to eight digits, with a gradual increase in difficulty. The total score for each task (direct or backward nonword recall) and classification according to age are used for statistical analysis.

\section{Corsi Block Test (CBT)}

The test is used to assess spatial memory and consists of a board on which nine blocks of equal dimensions are distributed in an irregular manner. During the evaluation, the examiner points at a sequence of blocks at the rate of one block per second and, after the end of the sequence, the subject will have to point out the blocks in the same sequence in which they were presented. The number of blocks of a sequence is increased gradually until the subject is no longer able to evoke the entire sequence. In the present study we used the protocol elaborated by Galera et al. ${ }^{20}$. The total score for the child (number of points when he remembered the exact order of the blocks plus the number of points when he remembered all blocks, but in the wrong order) was used for statistical analysis.

\section{Token Test - short version}

This test assesses comprehension by means of verbal commands. The original version of the test was presented by De Renzi and Vignolo in the early 60's and several adaptations were created thereafter. The Token Test - short version was presented by the same authors in $1975^{15}$.

The test involves 20 tokens of different sizes, colors and shapes distributed in a predetermined manner. The subject is asked to execute an order (e.g.: touch the red circle) and the examiner observes if he executes it exactly. The test consists of 13 tokens divided into 6 parts. Part 1 consists of seven tokens, parts 2, 3, 4 and 5 consist of four tokens, and part 6 of 13 tokens. In parts 1, 3 and 5, all tokens are used, while in parts 2, 4 and 6 only the large tokens are used. The level of complexity increases at each level, with level 5 consisting of more extensive sentences and level 6 also consisting of extensive orders which, however, require a greater cognitive question, i.e., a more complex 
attentional level (e.g.: touch the red circle if a blue circle is present).

The score is caculated by attributing 1 point to each item executed in a complete manner, ranging from 0 to 36 points. In this test we used the score for each level, the total score in the test and the classification of performance according to age ${ }^{21}$.

Data were analyzed statistically using the Student t-test for unpaired samples for the comparison of the numerical variable, and the equality of proportions test for between-group comparison of the number of children with a performance classfied as altered, with the level of significance set at $a=0.05$ for both tests.

\section{RESULTS}

Table 1 lists the absolute number of children and the percentages of patients classified as medium or inferior level for the RAVLT regarding the following items: immediate memory (A1), learning (sum of $A 1$ to $A 4)$, learning rate (A4-A1), retroactive interference (A5/ $A 4)$, long-term memory (A6), and recognition memory. There was a significant difference in retroactive interference $(p=0.002)$ and long-term memory $(p=0.01)$, with a higher percentage of children classified as showing inferior performance (altered) in G1.

Table 1. Absolute number and percentage of children classified as having inferior (altered) behavior or medium/superior performance in the various categories of the Rey Auditory-Verbal Learning Test (RAVLT)

\begin{tabular}{|c|c|c|c|c|c|c|c|c|c|}
\hline \multirow{3}{*}{ Tasks } & \multicolumn{4}{|c|}{ Group 1} & \multicolumn{4}{|c|}{ Group 2} & \multirow{3}{*}{$p$ - value } \\
\hline & \multicolumn{2}{|c|}{ Absolute Number } & \multicolumn{2}{|c|}{ Percentage \% } & \multicolumn{2}{|c|}{ Absolute Number } & \multicolumn{2}{|c|}{ Percentage \% } & \\
\hline & Inf & Med/sup & $\operatorname{lnf}$ & Med/sup & Inf & Med/sup & Inf & Med/sup & \\
\hline IM (A1) & 5 & 12 & 29 & 71 & 5 & 10 & 33 & 67 & 0.8 \\
\hline Learning & 5 & 12 & 29 & 71 & 3 & 12 & 20 & 80 & 0.5 \\
\hline Learning rate & 5 & 12 & 29 & 71 & 1 & 14 & 7 & 93 & 0.1 \\
\hline Retro. interf. & 12 & 5 & 71 & 29 & 1 & 14 & 7 & 93 & $0.002^{*}$ \\
\hline LTM & 8 & 9 & 47 & 53 & 1 & 17 & 7 & 93 & $0.01^{*}$ \\
\hline Recog. mem. & 7 & 10 & 41 & 59 & 2 & 13 & 13 & 87 & 0.08 \\
\hline
\end{tabular}

Equal proportion test - $(\alpha=0.05){ }^{*}=$ significant difference between groups

IM = immediate memory; Retro. interf. = retroactive interference; LTM = long-term memory/; Recog. mem. = recognition memory; Inf = inferior;

Med/sup = medium/superior

Table 2 lists the values obtained by Groups 1 and 2 in the Nonword and Digit Memory test (direct and backward order) and in the Corsi Block task. Table 3 presents the number of children with inferior or medium performance in each task. This analysis was not performed for the Corsi Block task since all children revealed inferior performance. Quantitative analysis revealed a significant difference between groups only for 3-syllable nonwords, with a better score/performance observed in Group 2. 
Table 2. Mean score obtained by Group 1 and Group 2 for the Nonword item with 2 syllables, 3 syllables, 4 syllables, 5 syllables and the total, in the direct order digit recall and backward digit recall tasks and the Corsi Block test

\begin{tabular}{cccccc}
\hline & \multicolumn{2}{c}{ Group 1 } & Group 2 & \multirow{2}{*}{ p - value } \\
\cline { 2 - 5 } & Score & SD & Score & SD & \\
\hline Pseudo - 2 syllables & 19.4 & 1.3 & 19.7 & 0.6 & 0.3 \\
Nonword - 3 syllables & 17.6 & 3.3 & 19.5 & 1.8 & $0.04^{*}$ \\
Nonword - 4 syllables & 16.9 & 4.7 & 18 & 3.7 & 0.4 \\
Nonword - 5 syllables & 13.5 & 6.9 & 16.9 & 4.2 & 0.1 \\
Nonword - Total & 67.6 & 13.9 & 74.4 & 8.1 & 0.1 \\
Digits - Direct order & 16 & 4.8 & 18.5 & 5.4 & 0.1 \\
Digits - Backward order & 6.3 & 3.5 & 6.3 & 3.2 & 0.9 \\
Corsi blocks & 4.1 & 2.3 & 4.3 & 2.2 & 0.8 \\
\hline
\end{tabular}

Student t-test for unpaired samples $(\alpha=0.05)$; ${ }^{*}=$ significant difference between groups

Nonword $=$ Nonwordwords; $\mathrm{SD}=$ standard deviation

Table 3. Absolute number and percentage per group of children classified with medium or inferior performance in the Nonword and Digit Memory Test

\begin{tabular}{ccccccccccc}
\hline \multirow{2}{*}{ Tasks } & \multicolumn{4}{c}{ Group 1 } & \multicolumn{4}{c}{ Group 2 } & \\
\cline { 2 - 9 } & \multicolumn{3}{c}{ Absolute Number } & Percentage \% & \multicolumn{1}{c}{ Absolute Number } & \multicolumn{2}{c}{ Percentage \% } & p - value \\
\cline { 2 - 9 } & Inf & Med/Sup & Inf & Med/Sup & Inf & Med/Sup & Inf & Med/Sup & \\
\hline Digits - direct order & 5 & 12 & 29 & 71 & 1 & 14 & 7 & 93 & 0.1 \\
Digits - backword order & 10 & 7 & 59 & 41 & 7 & 8 & 47 & 53 & 0.4 \\
Nonwords & 12 & 5 & 71 & 29 & 5 & 10 & 33 & 67 & 0.3 \\
\hline
\end{tabular}

Equal proportion test - $(\alpha=0.05){ }^{*}=$ significant difference between groups

Inf $=$ inferior; Med/sup $=$ medium/superior

Table 4 describes the values obtained for levels 1 to 6 of the Token Test - short version. It can be seen that the two groups showed similar scores, except for Level 6, for which Group 2 showed a significantly higher value (better performance). Comparison of the children with inferior performance revealed a normal pattern for 6 Group 1 participants (35\%) and 9 Group 2 participants (60\%), with no significant difference between groups $(p-$ value $=0.1)$.

Table 4. Mean and standard deviation (SD) of the scores for Group 1 and Group 2 participants in Levels 1 to 6 of the Token Test - short version

\begin{tabular}{ccccccc} 
& & Group 1 & & & Group 2 & \multirow{2}{*}{ p-value } \\
\cline { 2 - 5 } & Score & SD & Score & SD & \#\#\#\# \\
Level 1 & 7 & 0 & 7 & 0 & 0.5 \\
Level 2 & 3.9 & 0.3 & 3.8 & 0.4 & 0.1 \\
Level 3 & 3.9 & 0.3 & 3.5 & 0.9 & 0.9 \\
Level 4 & 3.5 & 0.6 & 3.5 & 0.9 & 0.5 \\
Level 5 & 3.1 & 1.1 & 2.9 & 1.1 & $0.02^{\star}$ \\
Level 6 & 6.5 & 2.8 & 8.5 & 2.2 & 0.2 \\
Total & 27.9 & 4.4 & 29.6 & 3.8 & \\
\hline
\end{tabular}

Student t-test for unpaired samples $(\alpha=0.05) ;{ }^{*}=$ significant difference between groups 


\section{DISCUSSION}

The objective of the present study was to compare memory, learning and oral comprehension aspects between two different samples of children: a group with a diagnosis of Attention Deficit Disorder and/ or Hyperactivity, and a group with Anxiety Disorder, diseases that commonly occur during childhood and represent risk factors for learning disorders.

Sample characterization revealed that $\mathrm{G} 1$ contained more boys than girls and a similar number of children classified as having predominant-inattentive ADHD or combined ADHD.

In the present study, there was homogeneity of ADHD subtypes. It should be pointed out that the groups were not subdivided for comparison of performance in cognitive skills since a recent study ${ }^{10}$ has reported that there is no relationship between ADHD subtypes and performance in tasks that measure working memory. In other words, the greater severity of the clinical behavior of ADHD subjects (inattentive, combined or impulsive) does not necessarily correspond to a worse performance in the tasks.

A balanced proportion of children in terms of sex and a greater prevalence of the separation anxiety disorder subtype were observed in G2. The literature reports that, in a clinical sample of the child population, the separation anxiety disorder is one of the most frequent in this age range and that separation anxiety disorder is similarly distributed between sexes in childhood ${ }^{2}$.

Regarding the cognitive skills considered in the present study, the first result was that the groups did not differ in working memory - visuospatial sketchpad, with this skill being impaired in both groups. According to the protocol used in the present study, the Corsi Block instrument not only requires a storage system for spatial coordinates, but the series of blocks should also be stored as a whole pattern of actions and hand movements. Thus, its execution involves both a memory system based on visuospatial coordinates and a kinesthetic system also able to store a sequence of movements.

The results obtained in the few studies that analyzed visuospatial working memory in children with ADHD ou $A D^{5,9,10}$ have revealed an inferior performance of these children in this skill. In populations of children at risk for delinquency, the symptoms of anxiety have been found to be positively correlated with difficulties in visual memory $^{5}$. Other studies, some of them also using the Corsi Blocks as done in the present study, have detected an inferior performance in ADHD children compared to control9,10. The same result was observed here among children with $A D$ and $A D H D$ and difficulties in working visuospatial memory.

In working memory - phonologic loop assessed by means of nonword repetition and direct and backword order digits there was a difference between groups in the repetition of three syllable nonwords, with a worse performance among G1 children. In general, in the nonword repetition task, $71 \%$ of G1 children were classified as having an altered performance, as opposed to $33 \%$ in G2. In a study that used the same instrument but on subjects of various age ranges ${ }^{22}$, the values detected in the 6-9 year age range (slightly younger than the range of the present study) were 19.5 for 2-syllable nonwords, 18.93 for 3-syllable nonwords, 16.33 for 4 -syllable nonwords, and 14.67 for 5 -syllable nonwords. These scores were similar to those for the $A D$ group, but were higher than those for G1. In the direct order digit task, most children of both groups exhibited medium performance $(71 \%$ for $\mathrm{G} 1$ and $93 \%$ for G2), and in the backward order, approximately half the children of both groups showed difficulty $(41 \%$ of and $53 \%$ of G2 ahowing medium performance).

Studies comparing working memory - phonologic loop between children with $A D H D$ and $A D$ reported that children with ADHD tended to have a worse performance in direct and backward digit order ${ }^{9,10,23}$, even though many children were classified as showing medium performance ${ }^{7}$.

Among the various types of stimuli, the most complex stimulus was represented by nonwords for children with $A D H D$ and by backward digit order for children with $A D$. The nonword stimulus is considered to be more complex than simply repeating digits in the direct order, since the storage of this information is not influenced by lexical, semantic or syntax factors ${ }^{24}$, with good auditory perception of the phonemes, as well as focused attention being also required in order to perform the task.

The backward digit subitem is also considered to be more complex since a sbject must store information and perform a complex activity for sequence inversion instead of simply repeating a sequence in the order presented $^{19}$. For this reason, a significant reduction of points is expected for backward order compared to direct order ${ }^{25}$.

Working memory performance by anxious individuals differs according to the type of task. Thoughts about irrelevant tasks, concerns, self-assessment and failure aspects may partially occupy the capacity of working 
memory, resulting in a reduction of competence in evoking information in more complex tasks. In contrast, in easy tasks the rest of working memory capacity would be sufficient to satisfy the demands of the task ${ }^{26}$. This statement may explain why AD children showed their worst performance in this task, since the literature reports that, among the tasks of nonword repetition and direct and backward digit repetition, the latter is the more complex ${ }^{19}$.

The RAVLT showed that most of the participants of the two groups were within normal limits for the first three items of the test: immediate memory (A1), learning ( $A 1$ to $A 4)$ and learning rate (A1 - A4), a fact suggesting adequate immediate verbal memory and learning ability of the participants.

The result for subitem A1 - immediate memory is positively related to performance in the task of direct order digit repetition. When comparing performance in the two tests (A1 and direct order digit repetition) a small and nonsignificant variability is expected. If the performance in $\mathrm{A} 1$ is much superior to performance in direct order digit repetition, this indicates that the child is inattentive or unmotivated ${ }^{27}$. In the present study, the percentage of $\mathrm{G} 1$ children with medium/superior performance in subitem A1 was identical to the percentage in the direct order digit task (71\%), showing coherence between tests. In contrast, in G2, the percentage of medium/superior performance was $93 \%$ for the direct digit ordering task, with this value being higher than the $67 \%$ value in subitem $A 1$ (incoherent performance). This result for G2 may suggest that some children with anxiety can suffer an initial negative impact when starting the task (involvement of an emotional question), although, when knowing how the task occurs, they become more tranquil and show improved performance ${ }^{26}$.

In the fourth item, retroactive interference (A5/A4), there was a significant difference between groups; $71 \%$ of $\mathrm{G} 1$ children were classified as below the mean, i.e., these children suffered retroactive activity, as opposed to only $7 \%$ of G2 children. Retroactive interference means that target information is forgotten due to the competition by new information. Thus, in the RAVLT, a child, when trying to memorize the stimulus words of the $B$ list (new words), are no longer able to remember the target words previously learned (words of the $A$ list) with a consequent difficulty in the memory consolidation of target words ${ }^{28}$. The present result (G1 children suffer retroactive interference) may be explained by the fact that children with difficulties in executive functions are more prone to inhibitory errors and therefore would be more susceptible to retroactive interference ${ }^{28}$.

In the fifth item of the test, retrieval (A6), there was also a significant difference between groups, although an improvement was observed in the performance of G1, in which $47 \%$ of the children were classified as having inferior performance. This finding suggests that some G1 subjects "organized" themselves in order to retrieve information that had previously suffered retroactive interference. Many of these subjects had initially learned the target stimuli; however, due to interference, there was no memory consolidation, with consequent impairment of their final performance. Regarding the recognition item, the number of children classified as being below normal levels remained similar to that for long-term memory in both groups, indicating that, even in the presence of "clues", the performance of children with memory difficulties did not improve.

The Token Test - short version investigates verbal comprehension and its complexity is due to the increased extension and complexity of the statements. In the present study, the score for the two groups was similar from level 1 to level 5 , whereas a significant difference between groups was observed for level 6 , with a worse score for G1. Levels 6 consists of more complex sentences, although they are not the most extensive. This level requires more attention than previous levels.

Although the Token test - short version is described as an instrument for the assessment of oral comprehension, several studies have stated that other cognitive skills are also required for this test ${ }^{29}$, such as working memory - phonologic loop, and linguistic and attention skills. For this reason, the other cognitive skills required, especially at level 6 , cause children with ADHD to exhibit a decline in performance. Mueller and Tomblin ${ }^{30}$ stated that children with ADHD properly understand superficial details, but have deficits in tasks that require a higher degree of effort and attention.

The total score obtained here by the ADHD group was 27.9, similar to the score reported by another Brazilian study that also used the Token Test - short version to assess oral comprehension in children with ADHD, although the children of the cited study were taking medication ${ }^{12}$. The authors of the study ${ }^{12}$ observed a difference between the ADHD group and the control group.

In general, both groups showed a poor performance in tests requiring verbal comprehension and working memory skills, whether the latter was stimulated 
verbally (nonwords or backward digits) or visually. However, it can be seen that $\mathrm{G} 1$ had a lower score than G2 in all tests (nonword repetition, direct and backward order digit repetition, RAVLT and Token test short version). The statistically significant difference between groups was demonstrated in task requiring the attentional aspects (retroactive interference, level six of the Token test - short version, and nonword repetition).

The importance of understanding the cognitive functioning of children with $A D H D$ and $A D$ should be one of the objectives of speech therapists, permitting an early diagnosis, the formulation of a therapeutic plan aiming at satisfying the needs of the child, as well as the development of compensatory strategies for these populations.

\section{CONCLUSION}

Children with ADHD exhibited more cognitive disorders than children with AD. The most complicated tasks for children with ADHD were the nonword repetition test, the oral comprehension of more extensive sentences and difficulties in memory consolidation in the presence of interference. In contrast, children with $A D$ had more difficulty in the backward digit recall test. The result "presence of retroactive interference" observed in the ADHD group, seems to be a good indicator that differentiates between children with ADHD and children with AD.

The present study demonstrated that there are some cognitive differences between these two conditions. Thus, speech professionals and audiologists should be alert to these changes, in oder to obtain an early diagnosis and plan an appropriate rehabilitation.

In the present study, specific cognitive skills, such as memory, learning and oral comprehension, were considered; however, further studies are needed for the investigation of other cognitive skills such as metalinguistic skills or executive functions and others, permitting the understanding of the cognitive profile of each neurdevelopmental disorder and of which skills differ among the various conditions. It is also necessary to analyze whether the cognitive performance of these groups is modified by some type of drug treatment and to determine the interference of cognitive rehabilitation regarding learning and cognition as a whole.

\section{REFERENCES}

1. Paterlini LSM. Triagem e diagnóstico de dificuldades de aprendizagem - Aplicação e desfecho de avaliações interdisciplinares de uma série de casos [Dissertação]. Ribeirão Preto (SP): Faculdade de Medicina, Universidade de São Paulo; 2017.

2. American Psychiatric Association - APA. Manual diagnóstico e estatístico de transtornos mentais DSM-5. Porto Alegre: Artmed; 2014.

3. Asbahr FR. Anxiety disorders in childhood and adolescence: clinical and neurobiological aspects. J. Pediatr. 2004;80(2):28-34. DOI: 10.1590/ S0021-75572004000300005.

4. Kusche CA, Cook ET, Greenberg WM. Neuropsychological and cognitive functioning in children with anxiety, externalizing, and comorbid psychopathology. J Clin Child Adolesc Psychol. 1993;22(2):172-95. DOI: 10.1207/ s15374424jccp2202_5.

5. Pine DS, Wasseman GA, Workman SB. Memory and anxiety in prepubertal boys at risk for delinquency. J Am Acad Child Adolesc Psychiatry. 1999;38(8):1024-31. DOI: 10.1097/00004583-199908000-00019.

6. Toren P, Sadeh M, Wolmer L, Eldar S, Koren S, Weizman $R$ et al. Neurocognitive correlates of anxiety disorders in children: a preliminary report. J Anxiety Disord. 2000;14(3):239-47. DOI: 10.1016/ S0887-6185(99)00036-5.

7. Naglieri JA, Goldstein S, Iseman JS, Schwebach A. Performance of children with attention deficit hyperactivity disorder and anxiety/depression on the WISC-III and cognitive assessment system (CAS). J. Psychoeduc. Assess. 2003;21(1):32-42. DOI: $10.1177 / 073428290302100103$.

8. Vasa RA, Nay RR, Klein RG, Mannuzza S, Moulton $\mathrm{JL}$, Guardino $\mathrm{M}$ et al. Memory deficits in children with and at risk for anxiety disorders. Depress Anxiety. 2007;24(2):85-94. DOI: 10.1002/da.20193.

9. Messina LF, Tiedmann KD. Avaliação da memória de trabalho em crianças com transtorno do déficit de atenção e hiperatividade. Psicol. USP. 2009;20(2):209-28.

10. Gallego-Martinez A, Garcia-Sevilla J, FenollarCortés J. Implicación de la memoria visoespacial y fonológica en la heterogeneidad clínica del Trastorno por Déficit de Atención con Hiperactividad (ADHD). An. Psicol. 2018;34(1):16-22. DOI: 10.6018/analesps.34.1.289671.

11. Vloet TD, Konrad K, Herpertz-Dahlmann B, Polier GG, Gunther T. Impact of anxiety disorders on attentional functions in children with ADHD. J 
Affect Disord. 2010;124(3):283-90. DOI: 10.1016/j. jad.2009.11.017.

12. Barini NS, Hage SRV. Vocabulary and verbal comprehension of students with Attention Deficit Hyperactivity Disorder. CoDAS. 2015;27(5):446-51. DOI: 10.1590/2317-1782/20152015022.

13. Baddeley AD. Working memory and language: an overview. J Commun Disord. 2003;36(3):189-208. DOI: 10.1016/S0021-9924(03)00019-4.

14. Serafini AJ, Fonseca RP, Bandeira DR, Parente MAMP. Panorama nacional da pesquisa sobre avaliação neuropsicológica de linguagem. Psicol. Cienc. Prof. 2008;28(1):34-49. DOI: 10.1590/ S1414-98932008000100004.

15. De Renzi E, Faglioni P. L'esame dei disturbi afasici di comprensione orale mediante una versione abbreviata del test dei gettoni. Riv Patol Nerv Ment. 1975;96:252-69.

16. Malloy-Diniz LF, Fuentes D, Abrantes SSC, Lasmar VAP, Salgado JV. Teste de aprendizagem auditivo-verbal de Rey - RAVLT. In: Malloy-Diniz LF, Fuentes D, Mattos P, Abreu N (orgs). Avaliação neuropsicológica. Porto Alegre: Artmed; 2010. p. 337-43.

17. Conselho Federal de Fonoaudiologia [internet]. Resolução CFFa no 466, de 22 de janeiro de 2015. Dispõe sobre as atribuições e competências relativas ao profissional Fonoaudiólogo Especialista em Neuropsicologia, e dá outras providências. [citado em janeiro de 2015]. Acessado em: http:// www.fonoaudiologia.org.br/cffa/wp-content/ uploads/2013/07/res-491-2016.pdf.

18. Oliveira RM, Fichman HC. Brazilian children performance on Rey's Auditory Verbal Learning Paradim. Arq Neuropsiquiatr. 2008;66(1):40-4. DOI: 10.1590/S0004-282X2008000100010.

19. Hage SRV, Grivol MA. Reference values of nonword repetition test for brazilian portuguese-speaking children. J. Appl. Oral Sci. 2009;17(spe):63-68. DOI: 10.1590/S1678-77572009000700011.

20. Galera C, Souza ALP. Memória visuoespacial e cinestésica de curto prazo em crianças de 7 a 10 anos. Estud. Psicol. 2010;15(2):137-43. DOI: 10.1590/S1413-294X2010000200002.

21. Malloy-Diniz LF, Bentes RC, Figueiredo PM, Brandao-Bretas D, da Costa-Abrantes S, Parizzi AM et al. Normalización de uma batería de tests para evaluar las habilidades de comprensión del lenguaje, fluidez verbal y denominación em niños brasileños de 7 a 10 años: resultados preliminares. Rev Neurol. 2007;44(5):275-80.

22. Grivol MA, Hage SRV. Phonological working memory: a comparative study between different age groups. J. Soc. Bras. Fonoaudiol. 2011;23(3):245-51. DOI: 10.1590/ S2179-64912011000300010.

23. Manassis K, Tannock R, Young A, John SF. Cognition in anxious children with attention deficit hyperactivity disorder: a comparison with clinical and normal children. Behav. Brain Funct. 2007;3(4):01-10. DOI: 10.1186/1744-9081-3-4.

24. Gathercole SE. Is nonword repetition a test of phonological memory or long-term knowledge? It all depends on the nonwords. Mem Cognit. 1995;23(1):83-94.

25. Freitas JOF, Aguiar CRR. Avaliação das funções cognitivas de atenção, memória e percepção em pacientes com esclerose múltipla. Psicol. Reflex. Crit. 2012;25(3):457-66. DOI: 10.1590/ S0102-79722012000300005.

26. Dutke S, Stober J. Test anxiety, working memory, and cognitive performance: supportive effects of sequential demands. Cogn Emot. 2001;15(3):381-9. DOI: 10.1080/02699930125922.

27. Lesak MD, Howieson D, Loring D. Neuropsychological assessment. Oxford: Oxford University Press; 2004.

28. Alves MVC, Bueno OFA. Retroactive interference: forgetting as an interruption of memory consolidation. Temas Psicol. 2017;25(3):1043-54. DOI: 10.9788/TP2017.3-07En.

29. Swihart AA, Panisset M, Becker JT, Beyer JR, Boiler $F$. The token test: validity and diagnostic power in Alzheimer's disease. Dev Neuropsychol. 1985;5(1):69-78. DOI: 10.1080/87565648909540423.

30. Mueller KL, Tomblin BJ. Examining the comorbidity of language disorders and ADHD. Top Lang Disorders. 2012;32(3):228-46. Sep 2012. DOI: 10.1097/TLD.0b013e318262010d. 